\title{
Corela
}

Cognition, représentation, langage

$13-2 \mid 2015$

Vol. $13, n^{\circ} 2$

\section{Impact de l'utilisation d'un support image dans la relation communicative chez les enfants avec autisme}

Sénia ALLAL

\section{OpenEdition}

Journals

Édition électronique

URL : http://journals.openedition.org/corela/4079

DOI : $10.4000 /$ corela.4079

ISSN : 1638-573X

Éditeur

Cercle linguistique du Centre et de l'Ouest - CerLICO

Référence électronique

Sénia ALLAL, « Impact de l'utilisation d'un support image dans la relation communicative chez les enfants avec autisme », Corela [En ligne], 13-2 | 2015, mis en ligne le 25 novembre 2015, consulté le 30 avril 2019. URL : http://journals.openedition.org/corela/4079; DOI : 10.4000/corela.4079

Ce document a été généré automatiquement le 30 avril 2019.

\section{(c) (i) (2)(2)}

Corela - cognition, représentation, langage est mis à disposition selon les termes de la licence Creative Commons Attribution - Pas d'Utilisation Commerciale - Partage dans les Mêmes Conditions 4.0 International. 


\title{
Impact de l'utilisation d'un support image dans la relation communicative chez les enfants avec autisme
}

\author{
Sénia ALLAL
}

1 Très tôt dans leur développement, les enfants qui s'inscrivent dans un processus d'évolution que nous dirons ordinaire, montrent une réciprocité sociale notamment dans leur conversation. Toutes les études spécialisées en la matière indiquent que vers huit mois, les bébés commencent à pointer le monde qui les environne et à identifier les choses. Ce qui implique que, dès l'âge d'un an, les enfants peuvent donc déjà bien communiquer avec leur entourage sans pour autant parler.

2 C'est après avoir pratiqué pendant quelques mois une communication facile, en l'occurrence le langage non verbal, que les enfants découvrent ce langage difficile, c'està-dire, la communication verbale. Une communication où nous ne voyons pas les mots, qui sont éphémères, et qui ouvre bon nombre de possibilités. Il va sans dire que le très jeune enfant communique déjà avec son entourage, mais ses premiers pas en ce domaine se font davantage de façon non verbale, autrement dit sans l'utilisation de mots. Il y a donc une abstraction du langage. Mais dès qu'il y a intention, il y a communication, et c'est le raffinement successif de ces intentions, en lien avec le développement des fonctions cognitives et articulatoires, qui mènera progressivement à l'apparition des premiers mots, autour du premier anniversaire de l'enfant.

3 Le langage est alors la fonction qui permet d'exprimer et de percevoir des états affectifs, des concepts, des idées, au moyen de signes. Il est présent dès la première année de la vie et accompagne pratiquement toutes les activités humaines. Son acquisition ne requiert pas d'effort spécial et, chez tout enfant vivant dans des conditions normales, une telle acquisition est attendue. Il suffit ainsi de penser à l'attente des parents qui se demandent quel sera le premier mot prononcé par l'enfant : papa ou maman? 
La notion de langage est purement théorique ; c'est l' "instance ", ou "faculté », qui est invoquée pour expliquer que tous les hommes se parlent. (Bronckart, 1997 : p7).

Néanmoins, cet aspect naturel et familier du langage est mis en question dès que l'enfant tarde à parler, mais aussi devant certains accidents qui touchent le langage installé, ou encore quand, du fait d'une pathologie, la communication s'altère, chose qui attire au premier degré notre attention. C'est pourquoi, d'emblée nous affirmerons qu'il serait probablement imprudent de dire des personnes qui ne partagent pas avec nous les codes communément admis par tous, qu'elles ne communiquent pas du tout. Dans cet ordre d'idée, nous pensons notamment au cas des enfants atteints d'autisme qui ne sont pas dans la même relation au monde et pour qui, indéniablement, la communication n'emprunte pas les canaux habituels. Nous nous proposons au cours de cette analyse d'en observer le comportement dans le cadre d'une réflexion qui portera sur la relation mode de communication / codes langagiers / perspectives de remédiation. Notre corpus sera donc essentiellement constitué d'enfants avec autisme.

5 Nous souhaitons, en effet, centrer notre travail justement sur ces difficultés et réfléchir sur des moyens qui permettraient éventuellement de rétablir la communication chez des personnes avec autisme. L'autisme est un trouble du développement humain caractérisé par une interaction sociale et une communication anormale, avec des comportements restreints et répétitifs.

6 Selon le neurolinguiste Léo Peeters, spécialisé dans les troubles du spectre autistique, l'autisme est une anomalie psychique complexe qui n'est plus considérée comme une maladie mentale mais comme un trouble du développement qui demande une prise en charge spécialisée. Une compréhension théorique de l'autisme et de ses conséquences est nécessaire si on veut intervenir de façon adaptée. (Peeters, 2008 : p229).

7 Les enfants avec autisme restent attachés au niveau de la perception. Dans ce cas, ils ne voient que ce qu'ils voient ; la signification sous-jacente est plus difficile à retrouver. C'est la raison pour laquelle, ils éprouvent aussi des difficultés avec les mots dans le langage verbal, car pour eux ces mots restent abstraits. Leur langage ne peut alors se développer qu'à partir d'éléments porteurs de sens, et concrétisés par des images / mots qui les ancreraient dans le contrat social.

Il a été prouvé que les enfants atteints d'autisme sont fréquemment attentifs à l'identification visuelle, même s'ils donnent l'impression de ne pas être soucieux de leur environnement. Ils en repèrent en tout cas les détails, compétence que nous nous proposons du reste d'exploiter en priorité dans les situations éducatives et pédagogiques. Le langage visuel est donc beaucoup plus facile à comprendre pour ces "penseurs visuels ", puisqu'ils ne comprennent que ce qu'ils voient et ne saisissent pas tout ce qui est abstrait, ils ne le perçoivent pas. L'utilisation d'un signe iconique présenterait donc de nombreux avantages: il est agréable à l'œil, source de motivation, et il facilite le processus de mémorisation et de compréhension. Ce qui va représenter une aide précieuse face au cas de ces enfants que nous tentons d'observer.

9 A cet effet, nous nous proposons donc d'inscrire notre projet d'analyse dans le recours à « l'image » fixe comme tremplin pour faciliter l'accès au sens, établir une communication avec le sujet choisi comme lieu d'observation, ainsi que pour multiplier les situations de communication. C'est ce qui explique que, si notre étude cherche à s'ancrer principalement dans le domaine de la didactique, elle sera, du fait de son objet, souvent tournée aussi vers la psycholinguistique à laquelle elle empruntera quelques-uns des 
outils qu'elle utilise et qui spécifient le domaine d'analyse et en facilitent l'accès. Ces outils-là nous sont nécessaires, non seulement au stade de l'observation, mais également au stade de la remédiation éventuelle. atteints d'autisme une altération qualitative des moyens de communication. Or, lorsque l'enfant n'acquiert pas normalement le langage verbal, ce qui est notamment leur cas, il nous a paru impératif de réfléchir à des moyens alternatifs et augmentatifs de la communication. Pour cela, nous avons eu recours à une méthode qui existe déjà, une méthode connue et expérimentée le PECS ${ }^{1}$, mais en essayant de l'implémenter dans notre propre terrain d'observation et d'en mesurer les effets et les lacunes éventuelles. Ce qui nous offrirait l'opportunité de proposer une démarche didactique à la fois adaptée aux cas des enfants observés et portant en elle les germes d'une généralisation possible à d'autres cas, sachant tout de même qu'une autre des caractéristiques de l'autisme est dans l'extrême variabilité des symptômes qui sont les siens.

La méthode PECS consiste essentiellement, s'agissant des enfants avec autisme, à remettre à l'interlocuteur l'image de l'objet qu'il désire obtenir en échange. Le PECS est donc un système de communication par échange d'images. Si la méthode PECS bénéficie d'une audience appréciable, c'est sans aucun doute en raison des résultats déjà obtenus en la matière, mais c'est aussi et surtout lié au fait qu'elle s'offre comme base à la fois possible et ouverte à tous les particularismes qui marquent justement le cas des enfants souffrant d'autisme. Ce qui élargit le champ d'observation de ces mêmes particularismes et l'adaptation de ce que la méthode propose à autant de variables que le terrain analysé peut nous donner à voir.

15 Nous tenterons donc d'observer le comportement de ces enfants en difficulté dans leur relation au support imagé. Nous tenons à préciser qu'il ne s'agit pas, dans ce cadre, de traiter de l'image en tant que telle mais de la valoriser pour son potentiel et son effet sur des apprenants ayant un dysfonctionnement de ce type en tant qu'outil permettant une meilleure mémorisation des signifiants et ainsi une augmentation de la production d'actes de communication.

16 Ce qui nous intéresse particulièrement dans cette étude, c'est de faire la preuve que le rapport langage-communication pourrait éventuellement s'inscrire dans des codes autres que ceux communément admis dans la plupart des cas qui désignent cette pathologie. 
Notre travail didactique c'est justement d'explorer la connaissance de ces codes et de les exploiter pour dégager éventuellement des lois générales qui permettraient une insertion sans douleur de ces enfants qui sont en dysfonctionnement et en marge de la société.

\section{Communicabilité / incommunicabilité}

17 A partir de cette thématique, qui est celle de la communication, et de la réception par rapport au schéma global de la communication, nous partons d'une base, celle du schéma de Jakobson, un schéma dans lequel un émetteur adresse un message à un récepteur à travers un canal qui leur permet d'établir et de maintenir la communication (Jakobson, 1963 : p214). Le message requiert un code, commun à l'émetteur et au récepteur. Ce qui nous interpelle dans le cas qui nous préoccupe c'est que c'est au niveau du canal que la communication s'altère, ce qui peut déjà expliquer que l'ambiguïté s'installe au cœur même de la communication entre ce qui est émis et ce qui est réceptionné chez les sujets observés. Cela va alors créer un domaine flou, une incompréhension et, par voie de conséquence, une communication qui s'effectue mal, voire qui ne se réalise pas du tout.

Notre domaine d'analyse vise à mettre en exergue, dans cette perspective, le rapport langage-communication chez les enfants atteints d'autisme, des enfants qui manifestent leur présence justement par cette absence de langage et par conséquent par l'absence de communication telle qu'elle est définie dans les codes généraux, normaux et donc habituellement admis par tous pour installer ce rapport.

Ce qui nous intéresse particulièrement, c'est d'abord cette rupture au niveau de la réception. Une rupture qui n'est pas totale puisque nous voudrions l'évoquer au moins par rapport à l'ambiguïté qui s'installe, et par rapport encore de façon plus générale et plus totale à celui de l'incompréhension qu'engendre cette ambiguïté. L'ambigüité s'installe au cœur même du message aussi bien au niveau de l'émission que de la réception ce qui fait que le message passe mal ou pas du tout, ce qui peut alors provoquer différentes réactions chez les enfants. Tout d'abord un calme excessif chez certains, qui réagissent à l'agression en se murant dans un silence et une solitude extrême, ignorant et fuyant ainsi toute communication, ou bien, à l'inverse, une agitation débordante, faite d'hurlements et de gestes divers, résultant d'un système de défense, ce qui va créer une incompréhension totale.

20 Nous partons pour cela du constat que les personnes qui souffrent d'un trouble autistique présentent des incapacités, si l'on se réfère aux travaux de l'Organisation Mondiale de la Santé, l'O.M.S.', plus au moins importantes dans les fonctions de communication, de socialisation, et d'imagination. Il en résulte bien entendu un désavantage social évident. Il s'agit là d'une pathologie, d'un dysfonctionnement du rapport langage-communication, parce que même lorsque les enfants souffrant d'autisme parlent, ils n'ont pas le même langage, la même codification, que nous.

21 Communiquer n'est pas toujours chose aisée, car de multiples obstacles peuvent bloquer l'échange, empêcher qu'il se réalise comme il serait souhaitable, et faire de l'incompréhension qui s'installe l'origine d'un conflit ou, pour le moins, d'une certaine incommunicabilité. La vie avec un enfant autiste est donc très dure. La vie de famille est vite perturbée par les problèmes de comportement de l'enfant, spécialement s'il développe une attitude agressive ou s'automutile. La famille vit dans une situation de stress chronique qui mine l'endurance des parents. 

élaboré grâce à la mobilisation et le dévouement de nombreux spécialistes, des didacticiens entre autres ainsi que des psychologues et des éducateurs. Toutes ces personnes nous ont aidées à élaborer ce protocole pour pouvoir réaliser cette recherche, nous nous sommes ainsi appuyée sur l'expérience de tous ces spécialistes ainsi que sur l'existence de plusieurs plans d'intervention comme la CIM10 ${ }^{3}, \mathrm{CARS}^{4}$ et l'ADOS 5 . Il s'agit donc d'une méthode combinée qui repose sur un ensemble d'éléments nous permettant ainsi d'atteindre nos objectifs.

\section{Présentation de la démarche} chaque enfant :

- Grille 1: (17 items) : habiletés de langage et compétences de communication. Âge de développement de 0 à 2 ans. 

personnes ou même des objets ou autre chose. Durant toutes ces activités l'enfant est assis sur une chaise devant une table et l'éducateur professionnel est assis sur une chaise devant lui à l'intérieur de la salle de classe. Nous proposons alors à l'enfant une série de pictogrammes correspondant aux objectifs à atteindre.

Grille 2- Habiletés de langage et compétences de communication. 


\begin{tabular}{|l|l|l|l|}
\hline Objectifs & Réponse & $\begin{array}{l}\text { Date } \\
\text { d'introduction }\end{array}$ & $\begin{array}{l}\text { Date } \\
\text { d'acquisition }\end{array}$ \\
\hline Habiletés réceptives & & & \\
\hline 1. Identifier des actions sur des images & 2 & $06-11-2012$ & $12-11-2013$ \\
\hline $\begin{array}{l}\text { 2. Identifier les objets selon leur fonction par les } \\
\text { images }\end{array}$ & 2 & $06-11-2012$ & $12-11-2012$ \\
\hline $\begin{array}{l}\text { 3. Identifier les émotions simples par les images } \\
\text { 4. Identifier les catégories, les familles par les } \\
\text { images }\end{array}$ & 1 & $06-11-2012$ & Non-acquis \\
\hline $\begin{array}{l}\text { 5. Répondre par oui ou non à des questions à } \\
\text { propos d'images ou d'objets }\end{array}$ & 1 & $06-11-2012$ & Non-acquis \\
\hline Habiletés expressives & $06-11-2012$ & Non-acquis \\
\hline 6. Exprimer le nom des objets par les images & 0 & $06-11-2012$ & Non-acquis \\
\hline $\begin{array}{l}\text { 7. Exprimer le nom des personnes familières par } \\
\text { les images }\end{array}$ & 0 & $06-11-2012$ & Non-acquis \\
\hline 8. Exprimer les actions sur les images & 0 & $06-11-2012$ & Non-acquis \\
\hline $\begin{array}{l}\text { 10. Demander verbalement les items } \\
\text { images }\end{array}$ & 0 & $06-11-2012$ & Non-acquis \\
\hline
\end{tabular}

\section{Analyse des données}

L'objectif est de permettre à D. d'accéder au savoir et à l'apprentissage. Concernant les habiletés réceptives, l'enfant a plutôt réussi à réaliser toutes les consignes.

Les deux premiers items sont totalement réussis. À ce stade là, le message passe.

\section{Item 1 : Identifier des actions sur des images}

Nous avons proposé à D. une série de quatre images. La première image représente un enfant en train de manger. La deuxième représente un petit garçon en train de jouer au ballon. La troisième représente une petite fille en train de s'habiller. Enfin, la quatrième représente un petit garçon en train de mettre ses chaussures.

Objectif : demander à l'enfant d'identifier chaque action sur les images.

Résultat : D. a obtenu un score de 2, ce qui correspond à un item totalement réussi. Il a parfaitement réalisé cet exercice en identifiant des actions sur des images. 

images.

\section{Item 5 : Répondre par oui ou non à des questions à propos d'images ou d'objets}

Nous avons proposé à $\mathrm{D}$. une série de quatre images qui représentent quatre objets différents. La première image représente un peigne, la deuxième une fourchette, la troisième un verre et enfin la quatrième un stylo.

Ensuite, dans une deuxième étape nous lui avons posé les questions suivantes tout en lui montrant l'image de l'objet. Les questions sont les suivantes: Est-ce qu'on se coiffe les cheveux avec une fourchette? Est-ce qu'on mange avec un peigne ? Est-ce qu'on boit avec un stylo? Est-ce qu'on écrit avec un verre? 

images.

\section{Item 9 : Exprimer les objets selon leur fonction par les images}

71 Nous avons proposé à D. une série de quatre images représentant des objets différents. La première représente une cuillère. La deuxième représente un peigne. La troisième représente un stylo. La quatrième représente du savon. 

fascinés par l'image, quel que soit l'objet, l'enjeu ou le discours qu'elle sous-tend. Nous pensons, de ce fait, qu'elle peut constituer un tremplin pour ces enfants leur permettant ainsi de s'exprimer autrement et d'avoir un mode de communication adéquat. L'image va aussi nous permettre d'aller vers ces enfants et de pénétrer dans leur monde en établissant ainsi des passerelles afin de pouvoir les pousser au maximum vers "notre monde ", et par là-même en leur permettant de se frayer un chemin vers un monde commun à tous. Il s'agit là d'un rapport de réciprocité. 
83 En outre, nous tenons à préciser l'étendue des apports bénéfiques que cela peut avoir sur la construction même de la personnalité de ces enfants. Car, même si l'handicap n'est pas totalement effacé, les enfants peuvent apprendre à construire leurs propres moyens de communication. En effet, nous avons pu constater au niveau de notre thèse qu'après en trainement et réévaluation: zéro à toutes les activités qu'on lui a proposées. En effet, D. n'est pas verbal ce qui explique qu'il est incapable de s'exprimer. Il est également incapable de formuler une demande. C'est ce qui va nous pousser alors à nous questionner en matière d'expression sur la possibilité de penser à autre chose de plus ou de différent que l'image. Quelque chose qui serait peut-être complémentaire du travail visé par l'image, c'est la raison pour laquelle au lieu de rester exclusivement sur l'image, on pourrait éventuellement penser à d'autres supports, à d'autres codes qui pourraient compléter ou renforcer le travail réalisé par l'image. Des codes autres tels que la musique, le dessin, la création d'ateliers qui se baseraient sur des travaux manuels en fonction des aptitudes qui sont développées par les enfants atteints d'autisme. Cela va permettre de rétablir le rapport langagecommunication chez ces derniers et ouvrant ainsi un bon nombre de perspectives. Nous mettons l'accent sur l'interrelation nécessaire entre plusieurs codes de communication afin de pouvoir obtenir les résultats voulus, ce qui donnera ainsi lieu à une réflexion didactique nouvelle ou même complémentaire.

éanmoins, nous sommes arrivée à la conclusion que si ces personnes ont du mal à communiquer avec «nous ", elles ne seront pas pour autant exclues de la société. Mais bien au contraire elles permettront, par leur difficulté-même à communiquer, d'élargir le champ de la communication et permettront éventuellement un enrichissement de celle-ci ainsi que des codes dans lesquels, il arrive souvent, qu'elles s'enferment. L'image va nous permettre alors d'avoir une meilleure compréhension de D. et donc d'établir un mode de communication commun. Le recours à l'image suppose en tout cas une mise en veille du langage des mots, la préférence étant donnée au visuel pour favoriser le développement langagier de ces enfants et par conséquent pour multiplier les situations de communication.

94 Ainsi, l'image-support- pour le langage et la communication serait un outil dont les variables extensibles à une infinité de cas ne feraient qu'adapter en l'amplifiant ce même outil aux besoins spécifiques de tous les enfants atteints d'autisme. 


\section{BIBLIOGRAPHIE}

Bronckart, Jean-Paul, 1997, Théories du langage, Bruxelle, Mardaga.

Hamm, Liliane, 1986, Lire des images, Paris, Armand Colin-Bourrelier.

Jakobson, Roman, 1963, Essais de linguistique générale, Paris, Minuit.

Joly, Martine, 2001, Introduction à l'analyse de l'image, France, Nathan.

Peeters, Theo, 2008, L'autisme, de la compréhension à l'intervention, Paris, Dunod.

\section{NOTES}

1. PECS, de l'anglais Picture Exchange Communication System, est un système de communication à base d'échange d'images.

2. La Classification Internationale des Handicaps est un manuel de classification des conséquences des maladies. Elaborée en 1980 sur l'initiative de l'OMS, la CIH doit l'essentiel de sa conception au professeur Philip Wood de l'Université de Manchester. Dans cet ouvrage, les concepteurs ont dégagé trois axes afin de définir au plus près la notion de handicap : Déficience, incapacité, désavantage.

3. CIM10 : Classification Internationale des Maladies.

4. CARS : Echelle d'évaluation diagnostic de l'autisme.

5. ADOS : Echelle d'observation pour le diagnostic de l'autisme.

6. Association Autisme Tlemcen.

7. Centre "Autisme Tlemcen" a vu le jour en février 2013 grâce à la mobilisation et la participation de l'Association Autisme Tlemcen.

8. Nous signalons que l'autisme est un trouble du développement qui touche quatre fois plus de garçons que de filles.

\section{RÉSUMÉS}

La communication véhicule un message qui passe par le langage. Ce qui n'exclut pas qu'elle puisse passer par d'autres codes. C'est pourquoi, il serait imprudent de dire des personnes qui ne 
partagent pas avec nous les codes communément admis, qu'elles ne communiquent pas. C'est le cas des autistes qui ne sont pas dans la même relation au monde que nous. Leur langage ne peut se développer qu'à partir d'éléments porteurs de sens et concrétisés par des images / mots qui les ancreraient dans le contrat social. Il sera question ici de créer un espace transversal à partir des codes développés par ces enfants et de la réception qu'on essaye d'en faire, donnant ainsi lieu à une création linguistique pour optimiser la communication.

The communication conveys a message that passes through language. This does not mean that it cannot pass through other codes. Therefore, it would be unwise to say that people who do not share with us the commonly accepted codes do not communicate. This is the case with persons with autism who are not in the same relationship to the world as we are. Their language can be developed only from meaningful elements and solidified by pictures/words that will anchor them in the social contract. We will discuss here creating a transverse space from the codes developed by these children and the reception we are trying to develop, giving rise to a linguistic creation in order to optimize communication.

\section{INDEX}

Mots-clés : Autisme, langage, communication, image, remédiation.

Keywords : Autism, language, communication, picture, remediation.

\section{AUTEUR}

\section{SÉNIA ALLAL}

Université Abou Bakr Belkaid, Tlemcen, Algérie

chaouiallal@yahoo.fr 